\title{
Orthopädische Versorgung in Europa
}

„Europa“ bestimmt mehr und mehr unser Handeln. Zahlreiche Gesetze für den nationalen Bereich werden von den Regelungen der Europäischen Region dominiert. Auch im Bereich der Medizin, und darunter der Orthopädie, wächst Europa zusammen. Dafür war der kürzlich in Brüssel veranstaltete Kongre $\beta$ der EFORT Beleg.

Anläßlich der Versammlung der Nationaldelegierten berichtete die orthopädische Sektion der Europäischen Vereinigung der Fachärzte (UEMS) über ihre Tätigkeiten und Ziele und legte interessante Datensammlungen zur orthopädischen Versorgungsstruktur in Europa vor. Die UEMS wurde 1958, 1 Jahr nach dem römischen Vertrag, gegründet. Ursprünglich hat sie das Ziel verfolgt, die medizinische Aus-, Weiter- und Fortbildung in Europa anzugleichen, so daß Ärzte in allen Ländern der europäischen Union ihren Beruf uneingeschränkt ausüben können. Seit 1962 gibt es Sektionen der UEMS für die wichtigsten Fachgebiete. Demgemäß gibt es auch eine orthopädische Sektion, die derzeit unter der Leitung von $M$. T. Speeckaert aus den Niederlanden steht und in der G. Fries aus Saarbrücken und $G$. Abt aus Berlin die deutschen Interessen vertreten. Inzwischen sind 34 europäische Staaten in der UEMS vertreten, darunter auch die Schweiz, obwohl sie nicht zur europäischen Union gehört. Das Interesse an Regelungen für einen gemeinsamen Weg in die Zukunft ist groß.

Die orthopädische Sektion hat Empfehlungen und Leitlinien zur inhaltlichen und strukturellen Harmonisierung der orthopädischen Weiterbildung in der europäischen Union publiziert. Diese sog ,minimal requirements for orthopaedic specialist training" machen klare Vorgaben bezüglich der Dauer und Qualität der orthopädischen Weiterbildung, die in dieser Form in Deutschland nicht vorliegen. G. Fries hat in einem Editorial an dieser Stelle bereits darauf hingewiesen, wie wichtig es sein wird, diese Vorgaben für die Orthopädie in der BRD zu berücksichtigen und umzusetzen (Fries 1997)

Inzwischen ist man dabei, auch Anforderungen für die Weiterbildungsstätten zu erarbeiten. Dabei wird durchaus berücksichtigt, daß die ärztliche und insbesondere orthopädische Weiterbildung in den einzelnen Ländern in unterschiedliche Systeme eingebettet ist. Für die BRD gilt allerdings, daß sie mit ihrem System der regional regulierten Weiterbildung fast einzigartig in Europa dasteht und hinsichtlich eines standardisierten Weiterbildungs- und Prüfungskonzeptes hinter vielen Ländern der Europäischen Union herhinkt.

Die orthopädische Sektion der UEMS bereitet darüber hinaus ein europäisches Examen vor und beschäftigt sich mit der kontinuierlichen medizinischen Fortbildung (CME), um die Qualität der orthopädisch-ärztlichen Versorgung hochzuhalten.

Von ganz besonderem Interesse sind die neuen Daten zur orthopädischen Versorgung in den Ländern der Europäischen Union (Tab. 1). Die Daten wurden durch Angaben der einzelnen Nationaldelegierten, aus den offiziellen Statistiken der Länder und nach Erhebungen der Industrie zusammengestellt. Es ist erstaunlich, wie unterschiedlich dicht die Versorgungsstrukturen in Europa sind. So gibt es

Z. Orthop. 137 (1999) 293-294

(C) 1999 Georg Thieme Verlag Stuttgart · New York in einem medizinisch durchaus nicht unterversorgten Land, wie den Niederlanden, nur 2,5 Fachorthopäden pro 100000 Einwohnern, in Spanien aber 5mal soviel, nämlich 12,8. Dabei nimmt die BRD mit 8,3 Orthopäden noch einen guten Mittelplatz ein.

Bei diesen Berechnungen sind Unterschiede der Systeme nicht berücksichtigt. So ist die Versorgungsdichte in Frankreich mit 3,3 Orthopäden/100000 Einwohner vergleichsweise gering. Allerdings gibt es dort den Rheumatologen, der die konservative Orthopädie mit vertritt. In Deutschland wiederum ist die Unfallchirurgie auf dem Gebiet der orthopädischen Chirurgie tätig. Würde man die Traumatologen in die Berechnung einbeziehen, ergäbe sich eine Versorgungsdichte von 12 Orthopäden und Traumatologen/100000 Einwohnern.

Die Ermittlung einer optimalen Versorgungsdichte ist wichtig, denn sie beeinflußt die Qualität der Versorgung und vorher schon die Qualität der Weiterbildung. Je größer die Zahl der Orthopäden, um so kleiner ist die Zahl der Operationen pro Spezialist und Jahr. In Europa fallen zwischen 6 (Griechenland) und 61 (Luxemburg) Gelenkersatzoperationen pro Spezialist an. Ist aber der luxemburgische orthopädische Chirurg deswegen der beste unter den Europäern, weil er in der Weiterbildung die meiste Erfahrung sammeln kann?

Welchen Einfluß hat die „Orthopädendichte“ auf die wünschenswerte qualitativ hochwertige (allerdings auch bezahlbare) Versorgung der Bevölkerung? Wie viele Orthopäden brauchen wir? - In Ländern mit wenigen Ärzten würde man Versorgungsdefizite erwarten. Aber ist die ärztliche Versorgung in diesen Ländern wirklich schlechter als in solchen mit hoher ärztlicher Versorgungsdichte? Dazu vermag Europa bisher keine Antwort zu geben. Zahlen aus den USA weisen anhand von sog. „outcome studies" darauf hin, daß eine hohe Versorgungsdichte nicht unbedingt mit besseren Behandlungsergebnissen einhergeht (Wennburg u. Cooper 1998, Weinstein et. al. 1998) Lee et al. (1998) haben in umfangreichen Untersuchungen einen Bedarf von 6 Orthopäden/100000 Einwohner für die USA ermittelt (die Traumatologie ist dabei eingeschlossen). „Which rate is right" fragt Weinstein im Journal of Bone and Joint Surgery, weil die Versorgungsdichte auch die Nachfrage stimuliert (supply-induced demand).

Dieser Frage werden sich auch die deutschen Fachgesellschaften und die UEMS annehmen müssen. Nachdem die Fachgesellschaften mit großem Aufwand Leitlinien zur Standardisierung der Prozeßqualität erstellt haben, steht nun auch die Strukturqualität zur Debatte.

\section{F. U. Niethard, Aachen}

\section{Literatur}

Fries, G.: Orthopädie und Europa - Editorial. Z. Orthop. 135 (1997) 477-478

Lee, P. P., C. A. Jackson, D. A. Relles: Demand-Based Assessment of Workforce Requirements for Orthopaedic Services. J. Bone Jt. Surg. 80-A (1998) 313-326

Weinstein, J., D. Goodman, J. E. Wennberg: The Orthopaedic Workforce: Which Rate is Right? J. Bone Jt. Surg. 80A (1998) 327-330 Wennburg, J., M. Cooper: The Dartmouth Atlas of Health Care. Chicago, American Hospital Publishing. 1998 
Tab. 1 Versorgungsstrukturen der Orthopädie in Europa (Quelle: UEMS 1999)

\begin{tabular}{|c|c|c|c|c|c|c|c|c|c|c|c|c|c|c|c|c|c|c|c|c|c|}
\hline & Austria & Belgium & $\begin{array}{l}\text { Switzer- } \\
\text { land }\end{array}$ & $\begin{array}{l}\text { Czech } \\
\text { Rep. }\end{array}$ & Germany & Denmark & Spain & France & U.K. & Greece & Hungary & Italy & Ireland & $\begin{array}{l}\text { Luxem- } \\
\text { bourg }\end{array}$ & Norway & Holland & Portugal & Poland & Romania & Sweden & Finland \\
\hline Population (million)1 & 8 & 10 & 7.1 & no data & 82 & 5.15 & 39 & 56 & 59 & 10.5 & no data & 5.6 & 3.5 & 0.4 & 4.5 & 15 & no data & no data & 23 & 8.8 & 5 \\
\hline Orthopaedic specialists & 560 & 720 & 467 & & 6845 & 286 & 5000 & 1860 & 1492 & 1100 & & 4000 & 71 & 20 & 250 & 380 & & & 418 & 600 & 246 \\
\hline Orthopaedic specialists/100000 population & 7 & 7.2 & 6.6 & & 8.3 & 5.6 & 12.8 & 3.3 & 2.5 & 10.5 & & 7.1 & 2 & 5 & 5.6 & 2.5 & & & 1.8 & 6.8 & 4.9 \\
\hline Population/Orthopaedic specialist & 14286 & 13889 & 15203 & & 11980 & 18007 & 7800 & 30108 & 39544 & 9545 & & 14000 & 49296 & 20000 & 18000 & 39474 & & & 55024 & 14667 & 20325 \\
\hline Orthopaedic specialists doing operations & 336 & 660 & 467 & & 2500 & 276 & 3000 & 1860 & 1492 & 1100 & & 2500 & 70 & 17 & 240 & 370 & & & 418 & 600 & majority \\
\hline Orthopaedic specialists treating trauma & 0 & 500 & $\sim 300$ & & unknown & 200 & 3000 & 1860 & 1145 & 1100 & & 2500 & 60 & 17 & 180 & 200 & & & 418 & 500 & majority \\
\hline Traumatologists & 100 & $?$ & 600 & & 2995 & 200 & 0 & $?$ & 33 & $?$ & & 0 & 0 & 46 & 400 & 800 & & & 0 & 0 & $?$ \\
\hline Private Orthopaedic specialists & 56 & 646 & 300 & & 4804 & 52 & $10 \%$ & 1120 & 166 & 200 & & 320 & 10 & 18 & 20 & 310 & & & 0 & 80 & 25 \\
\hline F/T salaried Orthopaedic specialists & 168 & 14 & 40 & & 2041 & 22 & $20 \%$ prac. & 370 & 1275 & 600 & & 1000 & 1 & 2 & 230 & 70 & & & 418 & 550 & \\
\hline Mixed salaried/private Orthopaedic specialists & 56 & 0 & 127 & & 135 & 30 & $70 \%$ & 370 & 1193 & 300 & & 2680 & 60 & 0 & 100 & 0 & & & very few & 0 & 221 \\
\hline Orthopaedic specialists $>60$ years & 56 & 25 & 32 & & 484 & 108 & & 160 & 152 & & & 600 & 3 & 2 & 30 & 50 & & & 43 & 57 & 14 \\
\hline Orthopaedic surgeons $51-60$ years & 140 & 148 & 141 & & 1993 & 131 & & 380 & 397 & & & 1280 & 34 & 3 & 90 & 100 & & & 105 & 275 & 67 \\
\hline Orthopaedic specialists $41-50$ years & 168 & 232 & 192 & & 2540 & 278 & & 740 & 579 & & & 1240 & 30 & 8 & 90 & 150 & & & 127 & 411 & 130 \\
\hline Orthopaedic specialists $\leq 40$ years & 196 & 315 & 102 & & 1531 & 17 & & 580 & 350 & & & 880 & 4 & 7 & 50 & 120 & & & 143 & 200 & 24 \\
\hline Hospital vacancies last year & 0 & 30 & 2 & & 0 & 30 & & $?$ & 121 & 80 & & 200 & 5 & 1 & 20 & 10 & & & 20 & 30 & $?$ \\
\hline Annual orthopaedic specialists acredited & 120 & 27 & 41 & & 280 & 32 & 149 & 60 & 108 & 250 & & 96 & 5 & 5 & 15 & 25 & & & 15 & 30 & 30 \\
\hline \% Annual orthopaedic specialists acredited/total specialists & 21 & 4 & 9 & & 4 & 11 & 3 & 3 & 7 & 23 & & 2 & 7 & 25 & 6 & 7 & & & 12 & 5 & 12 \\
\hline Official retirement age & 65 & 65 & 65 & & $65 / 68$ & $67 / 70$ & $65-70$ & 65 & 65 & 65 & & $65-72$ & 65 & 65 & 67 & 65 & & & 65 & 65 & 63 \\
\hline Number of hip replacement/year & 3000 & 13000 & 8000 & & 180000 & 5800 & 30000 & 60000 & 40000 & 4500 & & 40000 & 3076 & 915 & 5000 & 17000 & & & 3000 & 9500 & 4500 \\
\hline Number of knee replacements/year & 2000 & 4000 & 4000 & & 51000 & 2100 & 12000 & 20000 & 15000 & 1700 & & 8000 & 800 & 296 & 1000 & 600 & & & 600 & 5000 & 3500 \\
\hline Ratio total hip replacement/total knee replacement & 1.5 & 3.3 & 2 & & 3.5 & 2.8 & 2.5 & 3 & 2.7 & 2.6 & & 5 & 3.8 & 3.1 & 5 & 28.3 & & & 5 & 1.9 & 1.3 \\
\hline Annual total joints/specialist & 9 & 24 & 26 & & 34 & 28 & 8 & 43 & 37 & 6 & & 12 & 55 & 61 & 24 & 46 & & & 9 & 24 & 33 \\
\hline Total joint replacement/100000 population & 6250 & 17000 & 16901 & & 28171 & 15340 & 10769 & 14286 & 9322 & 5905 & & 8571 & 11074 & 30275 & 13333 & 11733 & & & 1565 & 16477 & 16000 \\
\hline W/L total hip - on average (weeks) & 30 & 4 & & & few & 20 & 24 & 8 & & 5 & & 60 & 26 & 3 & 20 & 16 & & & 4 & 52 & 20 \\
\hline W/L total hip - longest & 150 & 24 & 16 & & $3-6$ months & 125 & 100 & 24 & & 16 & & 85 & 102 & 4 & 52 & 50 & & & 110 & 72 & 150 \\
\hline W/L total hip-shortest & 2 & 2 & 2 & & 0 & 1 & 8 & 4 & & 1 & & 6 & 8 & 1 & 4 & 12 & & & 1 & 12 & 1 \\
\hline Trainee/surgical procedures/accreditation & yes & no & yes & & no & no & no & no & no & yes & & no & no & yes & yes & no & & & no & no & yes \\
\hline Working hours of: 1 - Trainees & no & no & no & & yes & yes & yes & yes & yes & yes & & no & yes & yes & yes & yes & & & no & yes & yes \\
\hline Working hours of: 2 -Specialists & no & no & no & & yes & yes & yes & yes & no & yes & & no & no & no & yes & no & & & no & yes & yes \\
\hline Recommended maximum hours? & 60 & 40 & 60 & & 38.5 & 37 & 40 & 40 & 72 & $42+$ & & 35 & 60 & 8 & 47 & 50 & & & no & 40 & 37 \\
\hline Applied to: 1 - Trainees & no & yes & no & & no & 37 & & no & yes & yes & & no & yes & yes & no & yes & & & no & yes & yes \\
\hline Applied to: 2 - Specialists & no & no & no & & no & 37 & & no & no & yes & & no & no & no & no & no & & & no & no & yes \\
\hline Number of training centres & 45 & 53 & 46 & & 235 & 5 & 80 & $?$ & 36 & 32 & & 25 & 21 & 2 & 60 & 12 & & & 10 & 40 & 5 \\
\hline Number of trainees & & $\sim 162$ & 204 & & $900-1000$ & 128 & 700 & ? & 871 & 250 & & 480 & 60 & 9 & 100 & 90 & & & 75 & 100 & 30 \\
\hline Annual output of trainees & & 35 & 27 & & 280 & 32 & 140 & 60 & 108 & 50 & & 96 & 5 & 1 & 15 & 25 & & & 15 & 30 & 6 -Sep \\
\hline Specification trainers/trainees & no & no & & & no & yes & yes & & no & no & & no & & no & yes & yes & & & no & no & no \\
\hline Ratio trainers/trainees & $1: 1$ & $1: 1.5$ & not know & & not known & $1: 1$ & & ? & not known & & & no & $1: 1.3$ & & $2: 1$ & $1: 1$ & & & 1.2 & not known & $?$ \\
\hline European Union Medical Trainees & 0 & 5 & not know & & not known & not known & 0 & ? & not known & & & 20 & 0 & 5 & 10 & 0 & & & 0 & a few & $? n$ \\
\hline Non European Medical Trainees & 0 & 15 & not know & & not known & no & 30 & $?$ & 304 & 40 & & 20 & 40 & 0 & 2 & 0 & & & 10 & a few & 4-Jan \\
\hline Immediate entry to training programme after graduation & no & no & no & & yes & no & & no, 2 & no & no & & yes & no & no & 0 & 2 to $3 y$ rs & & & yes & 1.9 year & no \\
\hline Common trunk & $2+$ & 2 & 2 & & 1 & 2 & & 2 & 2.5 & 1 & & 2 & 2 & 2 & 3 & 2 & & & 1 & spec. cont. & 5 \\
\hline Minimum Orthopaedic training before accreditation & 3.5 & 6 & 6 & & 5 & 5 & 5 & 6 & 6 & 5 & & 5 & 6 & 6 & 3 & 4 & & & 5 year & 5 years & 7 \\
\hline Minimum duration of Orthopaedic training before final exam & unspec. & 6 & 4 & & $5-6$ years & no exam & & 4 & 4 & 6 & & 5 & 4 & 6 & cont. assess & 4 & & & 5 year & 5 years & 1 \\
\hline Contentdduration of teaching? & yes & & yes & & yes & yes & yes & no & yes & yes & & no & yes & yes & yes & yes & & & yes & 5 years & yes \\
\hline$\%$ total specialists > 60 years & 25 & 21 & 30 & & 29 & 46 & 0 & 20 & 27 & 0 & & 32 & 48 & 15 & 36 & 26 & & & 25 & 46 & 27 \\
\hline$\%$ total specialists $51-60$ years & 30 & 32 & 41 & & 37 & 97 & 0 & 40 & 39 & 0 & & 31 & 42 & 40 & 36 & 39 & & & 30 & 69 & 53 \\
\hline$\%$ total specialists $41-50$ years & 35 & 44 & 22 & & 22 & 6 & 0 & 31 & 23 & 0 & & 22 & 6 & 35 & 20 & 32 & & & 34 & 33 & 10 \\
\hline$\%$ total speciaiists $\leq 40$ years & 0 & 4 & 0 & & 0 & 10 & 0 & & 8 & 7 & & 5 & 7 & 5 & 8 & 3 & & & 5 & 5 & \\
\hline
\end{tabular}

